Unfallchirurg $2020 \cdot 123: 2$

Online publiziert: 3. Dezember 2019

(c) Springer Medizin Verlag GmbH, ein Teil von Springer Nature 2019 https://doi.org/10.1007/s00113-019-00749-7

\section{Dank an die Gutachterinnen und Gutachter 2019}

Für die Qualität und Objektivität der Beiträge sind neben den engagierten Autorinnen und Autoren auch die vielen qualifizierten Gutachterinnen und Gutachter maßgeblich, die im Rahmen des Peer-Review-Prozesses die Manuskripte inhaltlich-wissenschaftlich prüfen und Empfehlungen zur konkreten Verbesserung äußern. Die Schriftleitung und die Redaktion von Der Unfallchirurg danken allen Gutachterinnen und Gutachtern, die im vergangenen Jahr Manuskripte für diese Zeitschrift begutachtet haben, sowie allen Leitthemenherausgebern herzlich für die konstruktive und gewissenhafte Arbeit. Durch ihren Einsatz garantieren sie die hohe Qualität und klinische Relevanz von Der Unfallchirurg. \author{
Christian Gerhardt, Karlsruhe \\ Oliver Gonschorek, Murnau \\ Steffen Ruchholtz, Marburg \\ Reinhard Hoffmann, Frankfurt am \\ Main \\ Sascha Flohé, Düsseldorf \\ Christian Willy, Ulm \\ Stefan Huber-Wagner, Schwäbisch Hall \\ Kajetan Klos, Jena \\ Ulrich Liener, Stuttgart
Iris Schleicher, Giessen \\ Ulrich Liener, Stuttgart
Iris Schleicher, Giessen \\ Tobias Helfen, München \\ Christian Kammerlander, M \\ Paul Grützner, Ludwigshafen
Frank Hildebrand, Aachen \\ Jens Geerling, Hannover
Julian Fürmetz, München \\ Florian Wolf, München \\ Florian Gebhard, Ulm \\ Elias Volkmer, München \\ Heiko Trentzsch, München
Christof Birkenmaier, München \\ Lars Peter Müller, Köln \\ Markus Muhm, Kaiserslautern \\ Johannes Bogner, München \\ Wolf Christian Prall, München
}

Fabian Stuby, Murnau Elisabeth Haas, München Stefan Grote, Straubing Simon Hackl, Murnau Arnold Suda, Mannheim Martin Langer, Münster Sebastian Wutzler, Wiesbaden Sebastian Fischer, Frankfurt am Main Dariusch Arbab, Dortmund Florian Früh, Zürich Georg Siebenbuerger, München Matthias Weuster, Kiel Jan Kuehle, Freiburg Moritz Crönlein, München Helmut Wegmann, Graz Stuart Hosie, München Franz Kralinger, Wien Oliver Miltner, Berlin

Karsten Labs, Berlin Bernhard Schwindl, Weiden Tobia Jung, Berlin Karl Thies, Greifswald Björn Rath, Aachen Marcus Jäger, Essen Joachim Andrassy, München Denis Ehrl, München Oliver Trapp, Murnau Tilman Calliess, Bern Oliver Gottschalk, München Frank Unglaub, Bad Rappenau
Franz Müller, Regensburg Bernd Kinner, Stuttgart Matthias Krause, Hamburg Tobias Schiergens, München Christian Ehrnthaller, München Sebastian Manegold, Berlin Johannes Zeichen, Minden Miriam Rüsseler, Frankfurt Dietmar Pennig, Köln Ernst Wiedemann, München Ingo Marzi, Frankfurt am Main Hans Gollwitzer, München Carl Neuerburg, München Markus Loibl, Zürich Matthias Knobe, Aachen Dominik Pförringer, München Karl-Heinz Frosch, Hamburg Simon Kargl, Linz

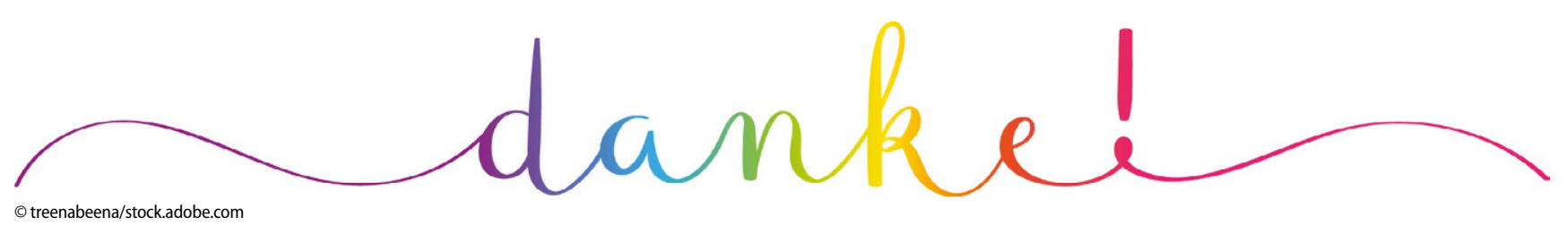

